Copyright $@ 2010$ Institute of Electrical and electronics Engineers, Inc.

All Rights reserved.

Personal use of this material, including one hard copy reproduction, is permitted.

Permission to reprint, republish and/or distribute this material in whole or in part for any other purposes must be obtained from the IEEE.

For information on obtaining permission, send an e-mail message to stds-igr@ieee.org.

By choosing to view this document, you agree to all provisions of the copyright laws protecting it.

Individual documents posted on this site may carry slightly different copyright restrictions.

For specific document information, check the copyright notice at the beginning of each document. 


\title{
Synchronization Criteria for Lur'e Complex Dynamical Networks with Coupling Delays
}

\author{
Ke Ding and Qing-Long Han*
}

\begin{abstract}
This paper is concerned with synchronization in Lur'e complex dynamical networks with coupling delays. Every identical node in the network can be represented as a Lur'e system. The influence of coupling delays on synchronization in networks is taken into account. Based on a Lur'e-Postnikov Lyapunov functional, some delay-dependant synchronization criteria are derived by employing a delay decomposition approach. A numerical example is given to illustrate the effectiveness of the synchronization criteria.
\end{abstract}

\section{INTRODUCTION}

Complex networks have received increasing attention from many fields of science and engineering due to their theoretical importance and practical applications. A complex network is a large set of interconnected nodes, in which a node is a fundamental unit with specific contents. Examples of complex networks include World Wide Web (WWW), electrical power grids, food webs, coauthorship and citation networks of scientists, business networks, and so on (See e.g., [2], [25]). Recent studies indicate that networks are characterized by three quantities: clustering coefficient, averaged path length, and degree distribution. One uses the clustering coefficient to measure the cliquishness of the typical neighborhood, and the clustering coefficient is proportional to the number of triangles contained in the network; one uses the averaged path length to measure the typical separation between two nodes in the network and the degree distribution to express the probability of finding a node having a definite number of edges. The small-world network and scale-free network are two classes of typical complex networks. A small-world network [25] is the network which has large clustering coefficient and short averaged path lengths. A scale-free network [2] is the network which has power law degree distribution.

There are two classes of distinct behaviors-static behaviors and dynamical behaviors within the complex networks. Undoubtedly, the dynamical behaviors are more complex and important than static ones. In order to well understand dynamical behaviors and to illustrate those behaviors mathematically, dynamical elements are introduced into all nodes

This work was supported in part by Central Queensland University for the Research Advancement Awards Scheme Project "Robust Fault Detection, Filtering and Control for Uncertain Systems with Time-Varying Delay" (Jan 2006 - Dec 2008).

Ke Ding is with the Centre for Intelligent and Networked Systems, and the School of Computing Sciences, Central Queensland University, Rockhampton, QLD 4702, Australia k. dingecqu. edu.au

Corresponding author: Qing-Long Han is with the Centre for Intelligent and Networked Systems, and the School of Computing Sciences, Central Queensland University, Rockhampton, QLD 4702, Australia; Tel. + 617 4930 9270; Fax. +61 74930 9729; q. han@ cqu.edu.au of complex networks which are described by the linearly coupled ordinary differential equations, and the existing complex network models are extended to dynamical network models. For the resulting complex dynamical networks, one of the most important dynamical behaviors is the synchronous motion of its nodes. In fact, synchronization is regarded as the collective behavior of self-organization which is a fundamental concept and universal phenomenon not only in daily life, such as fireflies flashing in unison, crickets chirping in synchrony, and heart cells beating in rhythm, but also in scientific areas, such as the synchronous transfer of digital or analog signals in chaotic systems for security communication and the synchronization of coupled oscillators (see e.g. [3], [5], [7] and [20]). Among the various analysis for synchronization in complex dynamical networks, the complete synchronization is the one focus of research, in which the state variables of individual systems converge to each other. Wu and Chua (1995) [27] discussed the synchronization in an array of linear couple dynamical systems. Li and Chen (2006) [16] studied the global synchronization in complex dynamical networks. Zhang et al. (2008) [30] considered the adaptive feedback synchronization in complex dynamical networks with delayed nodes. The existing synchronization results such as [9], [23], and [26] in complex dynamical networks are by means of linearizing the network at certain homogeneous state to analyze the master stability equations and thereby obtaining local synchronization results. This approach often requires to transfer the original network into the linear-varying network, and then to calculate the eigenvalues of outer-coupling matrix as well as the Jacobian matrix of a nonlinear function.

There are some nonlinear dynamical systems such as Chua's circuit, $n$-scroll attractors and hyperchaotic attractors [29] which can be expressed as Lur'e systems. This class of systems consists of a feedback connection of a linear dynamical system and a nonlinearity that satisfies sector condition [13]. During the last decade, the synchronization and stability criteria for Lur'e systems have been studied by using different methods such as employing Popov criteria and Kalman-Yacubovich-Popov (KYP) criteria (see e.g., [18], [19] and [21]), and constructing various Lur'e-Postnikovtype Lyapunov functionals to study the master-slave synchronization (see e.g., [1], [10], [13], [15], and [22]). Combining the theories of complex dynamical networks and Lur'e systems gives rise to a special complex dynamical networks, so-called Lur'e complex dynamical networks in which every identical node consists of a Lur'e system and all nodes interconnect and interact as the complex dynamical networks. 
It is easy to see that Lur'e complex dynamical networks not only inherit the feature from Lur'e systems, but also have the feature from complex dynamical networks. Therefore, it is of significant to study the dynamical behaviors of Lur'e complex dynamical networks, and to investigate how this class of complex dynamical networks can achieve the synchronization. Using Popov criteria, Liu et al. (2007) [17] studied the global synchronization in Lur'e complex dynamical networks.

Time delays are inevitable in the process of information transfer and have significant influences on the dynamical behavior of complex dynamical networks. Coupling delays which emerge from the process of all nodes' interaction are the feature of coupling parts of the whole networks. However, the synchronization results for Lur'e complex dynamical networks in [17] do not take into account time delays. To the best of authors' knowledge, there is no result available in the existing published literature to study Lur'e complex dynamical networks with coupling delays.

In this paper, we will introduce a class of Lur'e complex dynamical networks with coupling delays. Every node of this class of complex dynamical networks can be represented as a Lur'e system. We will investigate the effects of coupling delays on synchronization in Lur'e complex dynamical networks by employing a delay decomposition approach recently proposed by Han [12]. We will derive some delay-dependant synchronization criteria based on a Lur'e-Postnikov Lyapunov functional. In order to reduce the complexity of the criteria, we will avoid transferring the original network into the linear-varying network and calculating the eigenvalues of outer-coupling matrix as well as the Jacobian matrix of a nonlinear function. We will use a numerical example to illustrate the effectiveness of the synchronization criteria.

Notation: $\mathbb{R}^{n}$ denotes the $n$-dimensional Euclidean space. $\mathbb{R}^{m \times n}$ is the set of all $m \times n$ real matrices. For symmetric matrices $P$ and $Q$, the notation $P>Q$ (respectively, $P \geq Q$ ) means that matrix $P-Q$ is positive definite (respectively, positive semi-definite). $I_{N}$ is an identity matrix of $N \times N$ dimensions. $\operatorname{tr}(W)$ denotes the trace of matrix $W . \operatorname{diag}\left(a_{1}, a_{2}, \cdots, a_{n}\right)$ denotes the diagonal matrix. For an arbitrary matrix $W$ and two symmetric matrices $P$ and $Q$, the symmetric term in a symmetric matrix is denoted by *, i.e. $\left(\begin{array}{cc}P & W \\ * & Q\end{array}\right)=\left(\begin{array}{cc}P & W \\ W^{T} & Q\end{array}\right)$.

\section{PROBLEM STATEMENT}

Consider the Lur'e complex dynamical network with a coupling delay described by

$\left\{\begin{array}{l}\dot{x}_{i}(t)=A x_{i}(t)+B \varphi\left(C x_{i}(t)\right)+l \sum_{j=1}^{N} g_{i j} \Gamma x_{j}(t-\tau) \\ x_{i}(\theta)=\psi_{i}(\theta), \forall \theta \in[-\tau, 0], i=1,2, \cdots, N\end{array}\right.$

where $x_{i}=\left(x_{i 1}, x_{i 2}, \cdots, x_{i n}\right)^{T} \in \mathbb{R}^{n}$ is the state variables of unit $i$; the constant $l$ is the coupling strength; matrices $A=\left(a_{i j}\right)_{n \times n} \in \mathbb{R}^{n \times n}, B=\left(b_{i j}\right)_{n \times m} \in$ $\mathbb{R}^{n \times m}, C=\left(C_{1}^{T}, C_{2}^{T}, \cdots, C_{m}^{T}\right)^{T} \in \mathbb{R}^{m \times n} ; N$ is the amount of coupled nodes; $\tau>0$ is the constant time delay; $\psi_{i}(\theta)$ is a continuous vector-valued function; $\varphi\left(C x_{i}(t)\right)=$ $\left(\varphi_{1}\left(C_{1} x_{i}(t)\right), \varphi_{2}\left(C_{2} x_{i}(t)\right), \cdots, \varphi_{m}\left(C_{m} x_{i}(t)\right)\right)^{T}: \mathbb{R}^{m} \rightarrow$ $\mathbb{R}^{m}$ is a memoryless nonlinear vector-valued function which is globally Lipschitz, $\varphi(0)=0$, and suppose that the nonlinearity $\varphi(\cdot)$ is time invariant, decoupled, and satisfies a sector condition with $\varphi_{j}\left(C_{j} x_{i}(t)\right)$ belonging to a sector $\left[\underline{k}_{i}^{(j)}, \bar{k}_{i}^{(j)}\right]$, i.e., $\forall t \geq 0, \forall x_{i}(t) \in \mathbb{R}^{n}$,

$\left(\varphi_{j}\left(C_{j} x_{i}(t)\right)-\underline{k}_{i}^{(j)} C_{j} x_{i}(t)\right)\left(\varphi_{j}\left(C_{j} x_{i}(t)\right)-\bar{k}_{i}^{(j)} C_{j} x_{i}(t)\right) \leq 0$

$(i=1,2, \cdots, N ; j=1,2, \cdots, m)$. In the network (1), $\Gamma=$ $\left(\gamma_{i j}\right)_{n \times n} \in \mathbb{R}^{n \times n}$ is the constant inner coupling matrix of the nodes; $G=\left(g_{i j}\right)_{N \times N} \in \mathbb{R}^{N \times N}$ is the outer coupling matrix of the network and is defined as follows: if there is a connecting between node $i$ and node $j, j \neq i$, then $g_{i j}>0$; and the diagonal elements of $G$ are defined as

$$
g_{i i}=-\sum_{j=1, j \neq i}^{N} g_{i j}, i=1,2, \cdots, N .
$$

Compared with the requirements in [9], [23] which assumed that $G$ was symmetric and irreducible, and $g_{i j}=0$ or $g_{i j}=1, i \neq j, i, j=1,2, \cdots, N$, this paper has not those requirements for coupling matrix $G$.

We first introduce the following definition of synchronization in Lur'e complex dynamical networks with a single coupling delay.

Definition 1: The network (1) is said to achieve global asymptotical synchronization if

$$
\lim _{t \rightarrow \infty}\left\|x_{i}(t)-s(t)\right\|=0, i=1,2, \cdots, N
$$

where $\|\cdot\|$ represents the Euclidean norm, $s(t)$ is the solution of an isolate node and satisfies

$$
\dot{s}(t)=A s(t)+B \varphi(C s(t)), \quad s(0)=s_{0} .
$$

Remark 1: From Definition 1, it can be seen that as $t \rightarrow$ $\infty, x_{i}(t) \rightarrow s(t), i=1,2, \cdots, N$. In this situation, we use $x_{1}(t)=x_{2}(t)=\cdots=x_{N}(t)=s(t)$ to illustrate $x_{i}(t) \rightarrow$ $s(t)$. This kind of notion was used in [8], [14] and [24].

Let $e_{i}(t)=x_{i}(t)-s(t)$. From (1) and (4), we have

$$
\begin{aligned}
\dot{e}_{i}(t)= & A e_{i}(t)+B \kappa\left(C e_{i}(t) ; s(t)\right) \\
& +l \sum_{j=1}^{N} g_{i j} \Gamma e_{j}(t-\tau), i=1,2, \cdots, N
\end{aligned}
$$

where

$$
\begin{aligned}
& \kappa\left(C e_{i}(t) ; s(t)\right) \\
= & \left(\kappa_{1}\left(C_{1} e_{i}(t) ; s(t)\right) \cdots \kappa_{m}\left(C_{m} e_{i}(t) ; s(t)\right)\right)^{T} \\
= & \varphi\left(C x_{i}(t)\right)-\varphi(C s(t)) .
\end{aligned}
$$

Suppose $\kappa_{j}\left(C_{j} e_{i}(t) ; s(t)\right)$ belongs to the sector $\left[\underline{k}_{i}^{(j)}, \bar{k}_{i}^{(j)}\right]$, i.e., for $\forall t \geq 0, \forall e_{i}(t), s(t)$,

$$
\begin{aligned}
& \left(\kappa_{j}\left(C_{j} e_{i}(t) ; s(t)\right)-\underline{k}_{i}^{(j)} C_{j} e_{i}(t)\right) \\
& \times\left(\kappa_{j}\left(C_{j} e_{i}(t) ; s(t)\right)-\bar{k}_{i}^{(j)} C_{j} e_{i}(t)\right) \leq 0
\end{aligned}
$$

$(i=1,2, \cdots, N ; j=1,2, \cdots, m)$. Using the Kronecker product, the network (1) can be rewritten

$$
\left\{\begin{array}{l}
\dot{x}(t)=\tilde{A} x(t)+\tilde{B} \varsigma(\tilde{C} x(t))+H x(t-\tau) \\
x(\theta)=\tilde{\psi}(\theta), \quad \forall \theta \in[-\tau, 0]
\end{array}\right.
$$

where 


$$
\begin{gathered}
x(t)=\left(\begin{array}{cccc}
x_{1}(t) & x_{2}(t) & \cdots & x_{N}(t)
\end{array}\right)^{T}, \\
\varsigma(\tilde{C} x(t))=\left(\begin{array}{llll}
\varsigma_{1}\left(C x_{1}(t)\right) & \varsigma_{2}\left(C x_{2}(t)\right) & \cdots & \varsigma_{N}\left(C x_{N}(t)\right)
\end{array}\right)^{T}
\end{gathered}
$$

with $\varsigma_{i}\left(C x_{i}(t)\right)=\varphi\left(C x_{i}(t)\right)(i=1,2, \cdots, N), \tilde{\psi}(\theta)=$ $\left(\psi_{1}(\theta), \psi_{2}(\theta), \cdots, \psi_{N}(\theta)\right)^{T}$ is a continuous vector-valued function, and the system (5) can be rewritten as

$$
\dot{e}(t)=\tilde{A} e(t)+\tilde{B} \eta(\tilde{C} e(t) ; S(t))+H e(t-\tau)
$$

where

$$
\begin{gathered}
e(t)=\left(e_{1}(t) e_{2}(t) \cdots e_{N}(t)\right)^{T}, S(t)=(s(t) s(t) \cdots s(t))^{T}, \\
\eta(\tilde{C} e(t) ; S(t))=\left(\eta_{1}\left(C e_{1}(t) ; s(t)\right) \cdots \eta_{N}\left(C e_{N}(t) ; s(t)\right)\right)^{T}
\end{gathered}
$$

$(i=1,2, \cdots, N), \tilde{A}=I_{N} \otimes A, \tilde{B}=I_{N} \otimes B, H=l G \otimes$ $\Gamma, \tilde{C}=I_{N} \otimes C$. In order to define the initial condition of system (8), we supplement the initial value to $s(t)$ with $s(\theta)=s_{0}, \forall \theta \in[-\tau, 0)$. Consequently, the initial condition for system (8) is

$$
e(\theta)=\bar{\psi}(\theta), \forall \theta \in[-\tau, 0]
$$

where

$$
\bar{\psi}(\theta)=\left(\left(\psi_{1}(\theta)-s_{0}\right),\left(\psi_{2}(\theta)-s_{0}\right), \cdots,\left(\psi_{N}(\theta)-s_{0}\right)\right)^{T}
$$

is a continuous vector-valued function. Let

$$
\begin{aligned}
& \underline{K}=\operatorname{diag}\left(\underline{K}_{1}, \underline{K}_{2} \cdots, \underline{K}_{N}\right), \\
& \underline{K}_{i}=\operatorname{diag}\left(\underline{k}_{1}^{(i)}, \underline{k}_{2}^{(i)}, \cdots, \underline{k}_{m}^{(i)}\right) \in \mathbb{R}^{m \times m}, \\
& \bar{K}=\operatorname{diag}\left(\bar{K}_{1}, \bar{K}_{2}, \cdots, \bar{K}_{N}\right), \\
& \bar{K}_{i}=\operatorname{diag}\left(\bar{k}_{1}^{(i)}, \bar{k}_{2}^{(i)}, \cdots, \bar{k}_{m}^{(i)}\right) \in \mathbb{R}^{m \times m}, i=1,2, \cdots, N .
\end{aligned}
$$

Suppose that $\eta(\tilde{C} e(t) ; S(t))$ and $\varsigma(\tilde{C} x(t))$ belong to the sector $[\underline{K}, \bar{K}]$, i.e., for $\forall t \geq 0, \forall x(t), e(t), S(t)$,

$$
(\eta(\tilde{C} e(t) ; S(t))-\underline{K} \tilde{C} e(t))^{T}(\eta(e(t) ; S(t))-\bar{K} \tilde{C} e(t)) \leq 0,
$$

and

$$
(\varsigma(\tilde{C} x(t))-\underline{K} \tilde{C} x(t))^{T}(\varsigma(\tilde{C} x(t))-\bar{K} \tilde{C} x(t)) \leq 0 .
$$

It follows from Definition 1 that the global asymptotical stability of system (8), i.e., $\lim _{t \rightarrow \infty}\left\|e_{i}(t)\right\|=0(i=$ $1,2, \cdots, N)$, will ensure the global asymptotical synchronization in the network (1).

The purpose of this paper is to find sufficient conditions to ensure the global asymptotical synchronization in the Lur'e complex dynamical network with a coupling delay described by (1).

To end this section, we introduce some lemmas that will be used in the proofs of synchronization criteria.

Lemma 1: [4] (Schur Complement) For matrices $Q=$ $Q^{T}, S$ and $R=R^{T}$ of appropriate dimensions, the inequality $\left(\begin{array}{cc}Q & S \\ S^{T} & R\end{array}\right)>0$ holds, if and only if the following two inequalities hold $R>0, \quad Q-S R^{-1} S^{T}>0$.

An $\mathcal{S}$-procedure [28] plays an important role in absolute stability and robust stability theory. There are a number of variations, one of which is used in this paper can be expressed as follows.

Lemma 2: [4] Let $F_{i}=F_{i}^{T} \in R^{n \times n}, i=0,1,2, \cdots p$. Then the following statement is true $\epsilon^{T} F_{0} \epsilon>0$, for all $\epsilon \neq$ 0 satisfying $\epsilon^{T} F_{i} \epsilon \geq 0$ if there exist real scalars $\varepsilon_{i} \geq 0, i=$
$1,2, \cdots, p$ such that $F_{0}-\sum_{i=1}^{p} \epsilon_{i} F_{i}>0$. For $p=1$, these two statements are equivalent.

Lemma 3: [11] For any constant matrix $R>0, R=$ $R^{T} \in \mathbb{R}^{n \times n}$, scalar $\tau>0$, and vector function $e, \dot{e}$ : $[-\tau, 0] \rightarrow \mathbb{R}^{n}$ such that the following integrations are well defined, then

$$
-\int_{t-\tau}^{t} \dot{e}^{T}(s)(\tau R) \dot{e}(s) d s \leq\left(\begin{array}{c}
e(t) \\
e(t-\tau)
\end{array}\right)^{T}\left(\begin{array}{cc}
-R & R \\
R & -R
\end{array}\right)\left(\begin{array}{c}
e(t) \\
e(t-\tau)
\end{array}\right) .
$$

\section{SYNCHRONIZATION CRITERIA}

Since the nonlinearity $\varphi(\cdot)$ is time invariant and decoupled, in this section, we make use of this information to choose a Lur'e-Postnikov Lyapunov functional to obtain some synchronization criteria for the network (1).

We first study the case when $\varphi_{j}\left(C_{j} x_{i}(t)\right)$ and $\varsigma_{j}\left(C_{j} x_{i}(t)\right)$ belong to the sector $\left[0, \bar{k}_{i}^{(j)}\right]$, i.e., for $\forall t \geq 0, \forall x_{i}(t) \in \mathbb{R}^{n}$,

$$
\varphi_{j}\left(C_{j} x_{i}(t)\right)\left(\varphi_{j}\left(C_{j} x_{i}(t)\right)-\bar{k}_{i}^{(j)} C_{j} x_{i}(t)\right) \leq 0
$$

and

$$
\varsigma_{j}\left(C_{j} x_{i}(t)\right)\left(\varsigma_{j}\left(C_{j} x_{i}(t)\right)-\bar{k}_{i}^{(j)} C_{j} x_{i}(t)\right) \leq 0
$$

$(i=1,2, \cdots, N ; j=1,2, \cdots m)$. In this case, $\eta(\tilde{C} e(t) ; S(t))$ and $\varsigma(\tilde{C} x(t))$ belong to the sector $[0, \bar{K}]$, i.e., for $\forall t \geq 0, \forall e(t), S(t)$,

$$
\eta^{T}(\tilde{C} e(t) ; S(t))(\eta(e(t) ; S(t))-\bar{K} \tilde{C} e(t)) \leq 0 .
$$

and

$$
\varsigma^{T}(\tilde{C} x(t))(\varsigma(\tilde{C} x(t))-\bar{K} \tilde{C} x(t)) \leq 0 .
$$

Choose the following Lur'e-Postnikov Lyapunov functional

$$
\begin{aligned}
V\left(t, e_{t}\right)= & e^{T}(t) P e(t)+\sum_{i=1}^{M} \int_{t-i h}^{t-(i-1) h} e^{T}(\xi) Q_{i} e(\xi) d \xi \\
& +\sum_{i=1}^{M} \int_{-i h}^{-(i-1) h} \int_{t+\theta}^{t} \dot{e}^{T}(\xi)\left(h R_{i}\right) \dot{e}(\xi) d \xi d \theta \\
& +2 \sum_{i=1}^{N} \int_{0}^{C e_{i}(t)} \varsigma_{i}(s) d s
\end{aligned}
$$

where $e_{t}$ is defined as $e_{t}=e(t+\theta), \forall \theta \in[-\tau, 0]$, and $P \in \mathbb{R}^{N n \times N n}, P=P^{T}>0, Q_{i} \in \mathbb{R}^{N n \times N n}, Q_{i}=$ $Q_{i}^{T}>0, R \in \mathbb{R}^{N n \times N n}, R_{i}=R_{i}^{T}>0(i=1, \cdots, M)$; $h=\frac{\tau}{M}, M$ is the positive integer of division on the interval $[-\tau, 0]$ and $h$ is the length of each division. The integral term $\sum_{i=1}^{N} \int_{0}^{C e_{i}(t)} \varsigma_{i}(s) d s$ is well defined and nonnegative because $\varphi_{i}(s)$ is decoupled.

We now state and establish the following proposition.

Proposition 1: The error system described by (8), (13), and (14) is globally asymptotically stable, i.e., the Lur'e complex dynamical network with a coupling delay described by (1), (13) and (14) achieves global asymptotical synchronization, if there exist real matrices $P=P^{T}>0$, $Q_{i}=Q_{i}^{T}>0, R_{i}=R_{i}^{T}>0 \quad(i=1,2, \cdots, M)$, and $\Lambda=\operatorname{diag}\left(\lambda_{1}, \lambda_{2}, \cdots, \lambda_{N}\right)>0, \Xi=\operatorname{diag}\left(\xi_{1}, \cdots, \xi_{N}\right)>0$ such that

$$
\left(\begin{array}{cc}
\Delta^{(1)} & \Delta^{(2)} \\
\Delta^{(2) T} & \Delta^{(3)}
\end{array}\right)<0
$$


where

$$
\begin{aligned}
& \Delta^{(1)}=\left(\begin{array}{cc}
\Delta^{(1 \star)} & \Delta^{(2 \star)} \\
\Delta^{(2 \star) T} & \Delta^{(3 \star)}
\end{array}\right), \\
& \Delta^{(1 *)}=\left(\begin{array}{ccccccc}
\Delta_{11} & R_{1} & 0 & \cdots & 0 & P H & \Delta_{1} M+2 \\
* & \Delta_{22} & R_{2} & \cdots & 0 & 0 & 0 \\
* & * & \Delta_{33} & \cdots & 0 & 0 & 0 \\
\vdots & \vdots & \vdots & \ddots & \vdots & \vdots & \vdots \\
* & * & * & \cdots & \Delta_{M M} & R_{M} & 0 \\
* & * & * & \cdots & * & \Delta_{M+1} M+1 & H^{T} \tilde{C}^{T} \Xi \\
* & * & * & \cdots & * & * & -2 \Xi
\end{array}\right)
\end{aligned}
$$

with

$$
\begin{aligned}
& \Delta_{11}=P \tilde{A}+\tilde{A}^{T} P+Q_{1}-R_{1}, \\
& \Delta_{i i}=-R_{i-1}-R_{i}+Q_{i}-Q_{i-1} \quad(i=2,3, \cdots, M), \\
& \Delta_{1 M+2}=\tilde{A}^{T} \tilde{C}^{T} \Xi+\tilde{C}^{T} \bar{K} \Xi \text {, } \\
& \Delta_{M+1 M+1}=-Q_{M}-R_{M} \text {, } \\
& \Delta^{(2 \star)}=\left(\begin{array}{llll}
\Delta_{1 M+3} & 0 & \cdots & \tilde{B}^{T} \tilde{C}^{T} \Xi^{T}
\end{array}\right), \Delta^{(3 \star)}=-2 \Lambda, \\
& \Delta_{1 M+3}=P \tilde{B}+\tilde{C}^{T} \bar{K} \Lambda \text {, }
\end{aligned}
$$

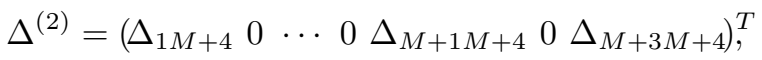

$$
\begin{aligned}
& \Delta^{(3)}=-\sum_{i=1}^{M} R_{i}, \Delta_{1 M+4}=h \tilde{A}^{T} \sum_{i=1}^{M} R_{i}, \\
& \Delta_{M+1 M+4}=h H^{T} \sum_{i=1}^{M} R_{i}, \Delta_{M+3 M+4}=h \tilde{B}^{T} \sum_{i=1}^{M} R_{i} .
\end{aligned}
$$

Proof: See the full version of the paper [6].

Remark 2: Notice that the system (8) is a Lur'e type retarded system. Proposition 1 provides a stability criterion. It is natural to compare Proposition 1 with some existing results in the literature. For example, when $M=1$, Proposition 1 reduces to Proposition 3 in [11]. Since the focus in this paper is the synchronization issue of Lur'e type complex dynamical network with a coupling delay, we will not further compare Proposition 1 with other absolute stability results.

Remark 3: It should be mentioned that for the first time, the Lur'e-Postnikov functional (15) is chosen to study the synchronization in the Lur'e complex dynamical networks with coupling delays.

For the nonlinearities $\eta(\tilde{C} e(t) ; S(t))$ and $\varsigma(\tilde{C} x(t))$ satisfying the more general sector condition (9), by using the loop transformation [13], we can conclude that global asymptotical stability of (8) in the sector $[\underline{K}, \bar{K}]$ is equivalent to that of the following system

$$
\dot{e}(t)=(\tilde{A}+\tilde{B} \underline{K} \tilde{C}) e(t)+\tilde{B} \tilde{\eta}(\tilde{C} e(t) ; S(t))+H e(t-\tau)
$$

in the sector $[0, \bar{K}-\underline{K}]$, where $\tilde{\eta}(\tilde{C} e(t) ; S(t))$ and $\varsigma(\tilde{C} x(t)))$ satisfy for $\forall t>0, \forall e(t), S(t)$,

$\tilde{\eta}^{T}(\tilde{C} e(t) ; S(t))(\tilde{\eta}(e(t) ; S(t))-(\bar{K}-\underline{K}) \tilde{C} e(t)) \leq 0$,

$$
\text { and } \left.{ }_{\varsigma^{T}}(\tilde{C} x(t))\right)(\varsigma(\tilde{C} x(t))-(\bar{K}-\underline{K}) \tilde{C} x(t)) \leq 0 .
$$

Then using Proposition 1, we have the following result.

Proposition 2: The error system described by (8), (9) and (10) is globally asymptotically stable, i.e., the Lur'e complex dynamical network with a coupling delay described by (1), (2) and (10) achieves global asymptotical synchronization, if there exist real matrices $P=P^{T}>0, Q_{i}=$ $Q_{i}^{T}>0, R_{i}=R_{i}^{T}>0(i=1,2, \cdots, M)$, and $\tilde{\Lambda}=$ $\operatorname{diag}\left(\tilde{\lambda}_{1}, \tilde{\lambda}_{2}, \cdots, \tilde{\lambda}_{N}\right)>0, \tilde{\Xi}=\operatorname{diag}\left(\tilde{\xi}_{1}, \tilde{\xi}_{2}, \cdots, \tilde{\xi}_{N}\right)>0$ such that

$$
\left(\begin{array}{cc}
\tilde{\Delta}^{(1)} & \tilde{\Delta}^{(2)} \\
\tilde{\Delta}^{(2) T} & \tilde{\Delta}^{(3)}
\end{array}\right)<0
$$

where

$$
\begin{gathered}
\text { where } \tilde{\Delta}^{(1)}=\left(\begin{array}{cccccc}
\tilde{\Delta}^{(1 \star)} & \tilde{\Delta}^{(2 \star)} \\
\tilde{\Delta}^{(2 \star) T} & \tilde{\Delta}^{(3 \star)}
\end{array}\right), \\
\tilde{\Delta}^{(1 \star)}=\left(\begin{array}{ccccccc}
\tilde{\Delta}_{11} & R_{1} & 0 & \cdots & 0 & P H & \tilde{\Delta}_{1 M+2} \\
* & \tilde{\Delta}_{22} & R_{2} & \cdots & 0 & 0 & 0 \\
* & * & \tilde{\Delta}_{33} & \cdots & 0 & 0 & 0 \\
\vdots & \vdots & \vdots & \ddots & \vdots & \vdots & \vdots \\
* & * & * & \cdots & \tilde{\Delta}_{M M} & R_{M} & 0 \\
* & * & * & \cdots & * & \tilde{\Delta}_{M+1} M+1 & H^{T} \tilde{C}^{T} \tilde{\Xi} \\
* & * & * & \cdots & * & * & -2 \tilde{\Xi}^{\prime}
\end{array}\right)
\end{gathered}
$$

with

$$
\begin{aligned}
& \tilde{\Delta}_{11}=P(\tilde{A}+\tilde{B} \underline{K} \tilde{C})+(\tilde{A}+\tilde{B} \underline{K} \tilde{C})^{T} P+Q_{1}-R_{1}, \\
& \tilde{\Delta}_{i i}=-R_{i-1}-R_{i}+Q_{i}-Q_{i-1}(i=2,3, \cdots, M), \\
& \tilde{\Delta}_{1 M+2}=(\tilde{A}+\tilde{B} \underline{K} \tilde{C})^{T} \tilde{C}^{T} \tilde{\Xi}+\tilde{C}^{T}(\bar{K}-\underline{K}) \tilde{\Xi}, \\
& \tilde{\Delta}_{M+1} M+1=-Q_{M}-R_{M}, \\
& \tilde{\Delta}^{(2 \star)}=\left(\tilde{\Delta}_{1} M+30 \cdots \tilde{B}^{T} \tilde{C}^{T} \tilde{\Xi}^{T}\right), \\
& \tilde{\Delta}^{(3 \star)}=-2 \tilde{\Lambda}, \tilde{\Delta}_{1} M+3=P \tilde{B}+\tilde{C}^{T}(\bar{K}-\underline{K}) \tilde{\Lambda}, \\
& \tilde{\Delta}^{(2)}=\left(\tilde{\Delta}_{1 M+4} 0 \cdots 0 \tilde{\Delta}_{M+1 M+4} 0 \tilde{\Delta}_{M+3}^{M+4}\right)^{T}, \\
& \tilde{\Delta}^{(3)}=-\sum_{i=1}^{M} R_{i}, \tilde{\Delta}_{1} M+4=h(\tilde{A}+\tilde{B} \underline{K} \tilde{C})^{T} \sum_{i=1}^{M} R_{i}, \\
& \tilde{\Delta}_{M+1} M+4=h H^{T} \sum_{i=1}^{M} R_{i}, \tilde{\Delta}_{M+3} M+4=h \tilde{B}^{T} \sum_{i=1}^{M} R_{i} .
\end{aligned}
$$

\section{A NumericAl EXAMPLE}

In this section, in order to illustrate the effectiveness of the synchronization criteria, we consider the complex dynamical network described by

$$
\begin{aligned}
\dot{x}_{i}(t)= & A x_{i}(t)+B \varphi\left(C x_{i}(t)\right) \\
& +l \sum_{j=1}^{5} g_{i j} \Gamma x_{j}(t-\tau), i=1,2, \cdots, 5
\end{aligned}
$$

where

$$
\begin{gathered}
A=\left(\begin{array}{cc}
-2 & 0 \\
0 & -0.9
\end{array}\right), B=\left(\begin{array}{c}
-0.2 \\
-0.3
\end{array}\right), C=\left(\begin{array}{cc}
0.6 & 0.8
\end{array}\right), \\
\Gamma=\left(\begin{array}{ll}
1 & 0 \\
0 & 1
\end{array}\right), G=\left(\begin{array}{ccccc}
-4 & 1 & 1 & 1 & 1 \\
1 & -1 & 0 & 0 & 0 \\
1 & 0 & -1 & 0 & 0 \\
1 & 0 & 0 & -1 & 0 \\
1 & 0 & 0 & 0 & -1
\end{array}\right),
\end{gathered}
$$

and $l=1, \varphi(\xi)=1 / 2(|\xi+1|-|\xi-1|), \varphi(\xi)$ belongs to a sector $[\underline{K}, \bar{K}]$ with $\underline{K}=0$ and $\bar{K}=1$. By Kronecker product and Definition 1, one can obtain the following error system

$$
\dot{e}(t)=\tilde{A} e(t)+\tilde{B} \eta(\tilde{C} e(t) ; S(t))+H e(t-\tau)
$$

where $\tilde{A}=I_{5} \otimes A, \tilde{B}=I_{5} \otimes B, \tilde{C}=I_{5} \otimes C, H=$ $G \otimes \Gamma$. Applying Proposition 1 to this example, the maximum 


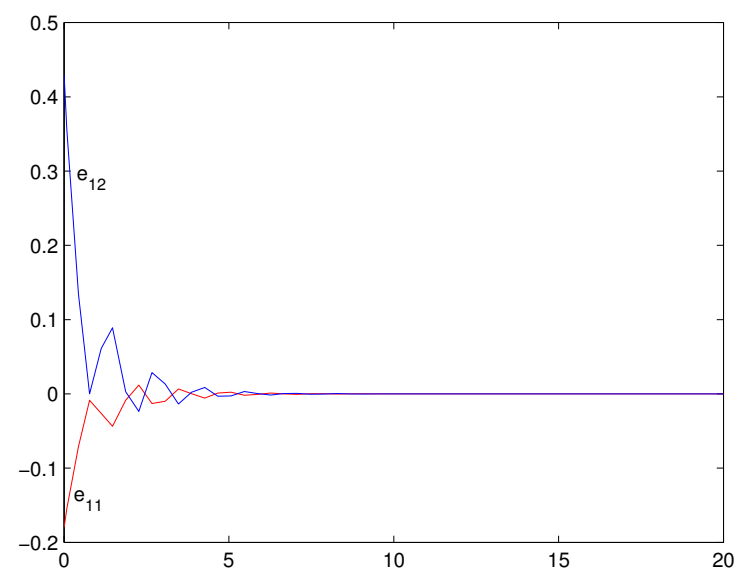

Fig. 1. The synchronization errors $e_{11}$ and $e_{12}$ of (22) corresponding to node 1 of (21) with the coupling delay $\tau=0.3793$

allowed coupling delay bound $\tau_{\max }$ is calculated for different $M$, and the results are listed in Table I. From this table, one can also see that the larger $M$, the larger $\tau_{\max }$.

TABLE I

THE MAXIMUM ALLOWED COUPLING DELAY BOUND FOR DIFFERENT $M$

\begin{tabular}{|c|c|c|c|c|c|}
\hline $\mathrm{M}$ & 1 & 2 & 3 & 4 & 5 \\
\hline$\tau_{\max }$ & 0.3310 & 0.3676 & 0.3749 & 0.3778 & 0.3793 \\
\hline
\end{tabular}

Taking the coupling delay as $\tau=0.3793$, we depict the simulation results for error variables $e_{i j}$ of (22) in Fig. 1 to Fig. 5, respectively, where the initial conditions as $\left(e_{11}, e_{12}\right)=(0.1 ;-0.2),\left(e_{21}, e_{22}\right)=(0.1 ;-0.2)$, $\left(e_{31}, e_{32}\right)=(0.1 ;-0.2),\left(e_{41}, e_{42}\right)=(0.1 ;-0.2)$ and $\left(e_{51}, e_{52}\right)=(0.1 ;-0.2)$, from which one can clearly see that the system (22) is globally asymptotically stable, i.e., the complex dynamical network (21) indeed achieves synchronization.

Now, we consider Lur'e complex dynamical networks (21) with $\underline{K}=0.2$ and $\bar{K}=0.8$. Using Proposition 2 to this case, the maximum allowed coupling delay bound $\tau_{\max }$ is calculated for different $M$, and the results are listed in Table II. From this table, one can also see that the larger $M$, the larger $\tau_{\max }$. Simulation results are omitted due to page limit.

TABLE II

THE MAXIMUM ALLOWED COUPLING DELAY BOUND FOR DIFFERENT $M$

\begin{tabular}{|c|c|c|c|c|c|}
\hline $\mathrm{M}$ & 1 & 2 & 3 & 4 & 5 \\
\hline$\tau_{\max }$ & 0.3368 & 0.3734 & 0.3807 & 0.3836 & 0.3840 \\
\hline
\end{tabular}

\section{CONCLUSIONS AND FUTURE WORKS}

Synchronization in Lur'e complex dynamical networks with single coupling delay have been addressed. Some synchronization criteria of the networks have been derived based on a Lur'e-Postnikov Lyapunov functional. In order to reduce

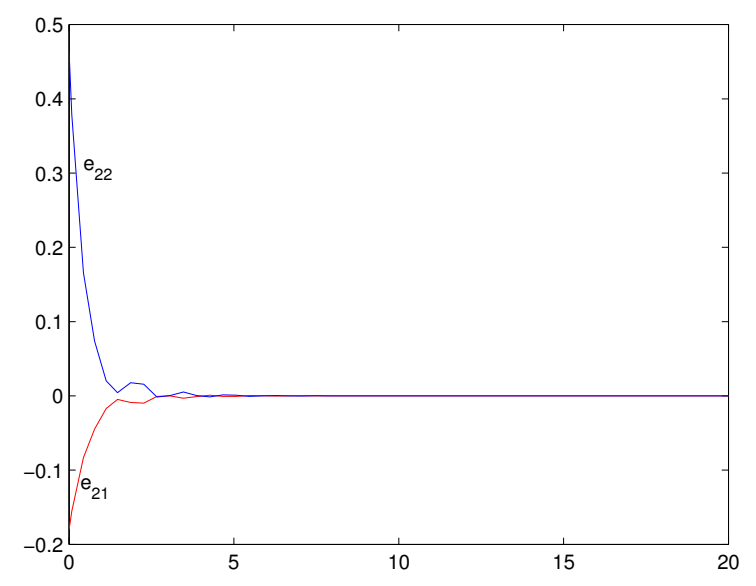

Fig. 2. The synchronization errors $e_{21}$ and $e_{22}$ of (22) corresponding to node 2 of (21) with the coupling delay $\tau=0.3793$

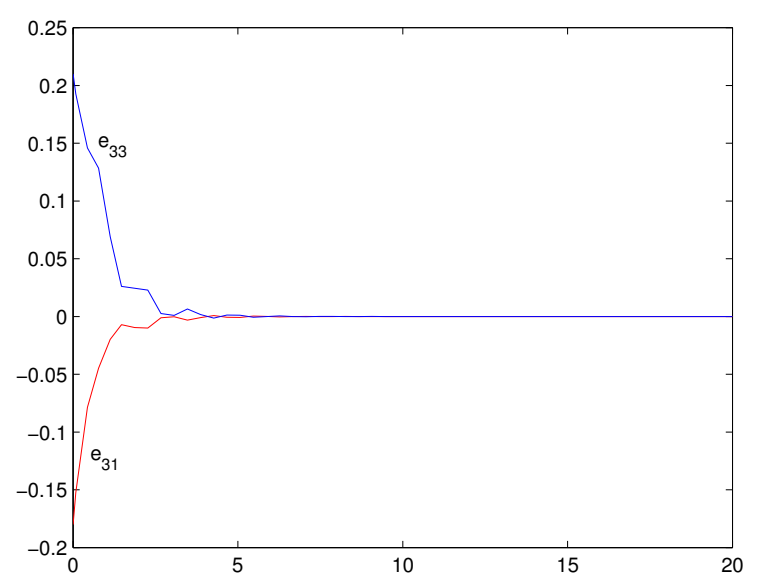

Fig. 3. The synchronization errors $e_{31}$ and $e_{32}$ of (22) corresponding to node 3 of (21) with the coupling delay $\tau=0.3793$

the complexity of the criteria, we have avoided transferring the original network into the linear-varying network and calculating the eigenvalues of the outer-coupling matrix as well as the Jacobian matrix of a nonlinear function. And we have employed the delay decomposition approach which can provide the larger maximum allowed coupling-delay bound of complex dynamical networks as the integer of division for the delay interval increases. The effectiveness of the synchronization criteria has been proved through a numerical example.

It should be noted that in this paper we just consider a single delay in the coupling part of the Lur'e complex dynamical network (1). The synchronization in the Lur'e complex dynamical network (1) with multiple coupling delays and time-vary coupling delays both in every node and in the whole coupling part are still worthwhile to be investigated. We will conduct such study in the future work. 


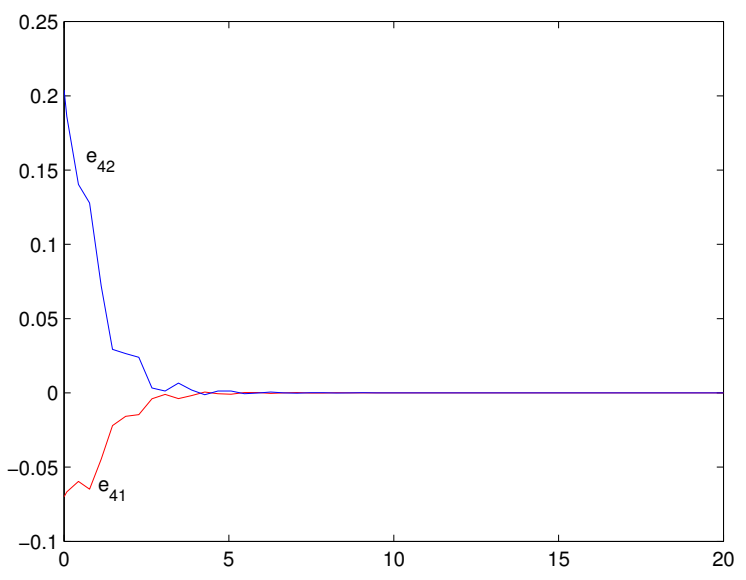

Fig. 4. The synchronization errors $e_{41}$ and $e_{42}$ of (22) corresponding to node 4 of (21) with the coupling delay $\tau=0.3793$

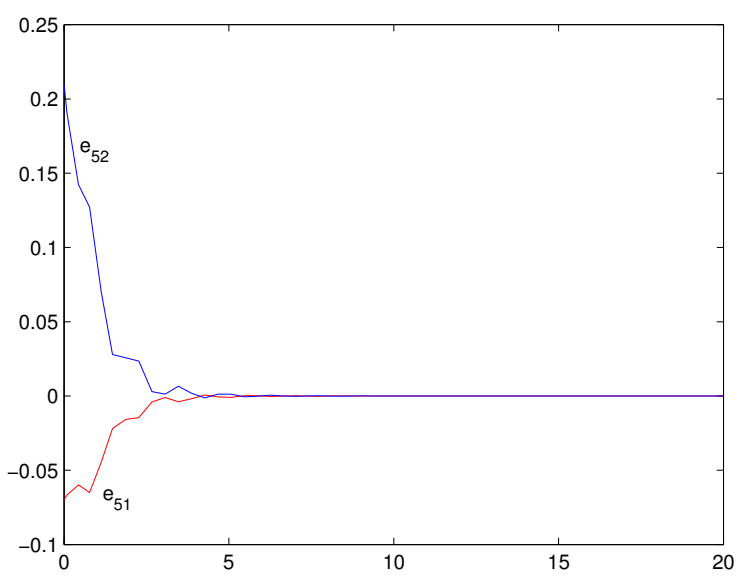

Fig. 5. The synchronization errors $e_{51}$ and $e_{52}$ of (22) corresponding to node 5 of (21) with the coupling delay $\tau=0.3793$

\section{REFERENCES}

[1] M. A. Aizerman and F. R. Gantmacher, Absolute stability of regulator systems, San Francisco, Holden-Day, CA; 1964.

[2] A. L. Barabasi and R. Albert, "Emergence of scaling in random networks," Science, vol. 286, no. 5439, 1999, pp. 509-512.

[3] M. V. L. Bennett and R. S. Zukin, "Electrical coupling and neuronal synchronization in the mammalian brain," Neuron, vol. 41, no. 4, Feb. 2004, pp. 495-511.

[4] S. Boyd, L. EI Ghaoui, E. Feron, and V. Balakrishnan, Linear Matrix Inequalities in Systems and Control Theory, Philadephia, SIAM, PA; 1994.

[5] P. F. Curran and L. O. Chua, "Absolute stability theory and the synchronization problem," Int. J. Bifurcat. Chaos, vol. 7, no. 6, 1987, pp. 1375-1383.

[6] K. Ding and Q.-L. Han, "Synchronization Criteria for Lur'e Complex Dynamical Networks with Coupling Delays," The full version of this conference paper.

[7] H. Fujisaka and T. Yamada, "Stability theory of synchronization motion in coupling-oscillators systems," Proced. Theor. Phys., vol. 69, no. 1, 1983, pp. 32-47.

[8] J. Fan, X. Li, and X. Wang, "On synchronous preference of complex dynamical networks," Physica A, vol. 355, no. 1, 2005, pp. 657-666.
[9] H. J. Gao, J. Lam, and G. Chen, "New criteria for synchronization stability of general complex dynamical networks with coupling delays," Phys. Lett. A, vol. 360, 2006, pp. 263-273.

[10] W. M. Haddad and V. Kapila, "Absolute stability criteria for multiple slope-restricted monotonic nonlinearities," IEEE Trans. Automat. Contr., vol. 40, Feb. 1995. pp. 361-365.

[11] Q.-L. Han, "Absolute stability of time-delay systems with sectorbounded nonlinearity," Automatica, vol. 41, no. 12, 2005, pp. 21712176.

[12] Q.-L. Han, "A delay decomposition approach to stability and $H_{\infty}$ control of linear time-delay system-Part I: Stability," Proceedings of the 7th World Congress on Intelligent Control and AutomationWCICA'08, Chongqing, China, 2008, pp. 289-294.

[13] H. K. Khalil, Nonlinear systems, Upper Saddle River, Prentice-Hall, NJ; 1996.

[14] C. P. Li, W. G. Sun, and J. Kurths, "Synchronization of complex dynamical networks with time delays," Physica A, vol. 361, 2006, pp. 24-34.

[15] X. Liao, Absolute stability of nonlinear control systems, Science Press, Beijing; 1993.

[16] Z. Li and G. Chen, "Global synchronization and asymptotic stability of complex dynamical networks," IEEE Trans. Circuits Syst. II, Exp. Briefs, vol. 53, no. 1, Jan. 2006, pp. 28-33.

[17] X. Liu, J. Z. Wang, and L. Huang, "Global synchronization for a class of dynamical complex networks," Physica A, vol. 386, no. 1, 2007, pp. 543-556.

[18] V. M. Popov, Hyperstability of control systems, New York, Springer, NY; 1973.

[19] V. M. Popov, "Absolute stability of nonlinear systems of automatic control," Avtomatika i Telemekhanika, vol. 22, 1961, pp. 961-979.

[20] L. M. Pecora and T. L. Carroll, "Synchronization in chaotic systems,' Phys. Rev. Lett., vol. 64, no. 8, 1990, pp. 821-824.

[21] A. Rantzer, "On the KalmanYakubovichPopov lemma," Syst. Control Lett., vol. 28, no. 1, 1996, pp. 7-10.

[22] A. Somolines, "Stability of Lurie type functional equations," J. Differ Equations, vol. 26, 1977, pp. 191-199.

[23] X. Wang and G. Chen, "Synchronization in general complex dynamical networks with coupling delays," Physica A, vol. 343,2004, pp. 263-278.

[24] L. Wang, H. P. Dai, and Y. X. Sun, "Synchronization criteria for a generalized complex delayed dynamical network model," Physica A, vol. 383, 2007, pp. 703-713.

[25] D. J. Watts and S. H. Strogatz, "Collective dynamical of 'small-world' networks," Natures, vol. 393, 1998,pp. 440-442.

[26] C. W. Wu, Synchronization in complex networks of nonlinear dynamical systems, World Scientific Publishing, Singapore; 2007.

[27] C. W. Wu and L. O. Chua, "Synchronization in an array of linear coupled dynamical systems," IEEE Trans. Circuits and Syst.-I, vol. 42, no. 8, Aug. 1995, pp. 430-447,

[28] V. A. Yakubovich, G. A. Leonov, and A. Kh. Gelig, Stability of stationary sets in control systems with discontinuous nonlinearities, World Scientific, Singapore; 2004.

[29] M. E. Yalcin, J. A. K. Suykens, and J.Vandewalle, "Master-slave synchronization of Lur'e systems with time-delays," Int. J. Bifurcat. Chaos, vol. 11, no. 6, 2001, pp. 1707-1722.

[30] Q. J. Zhang, J. A Lu, and J. H. Lü, "Adaptive feedback synchronization of a general complex dynamical network with delayed nodes," IEEE Trans. Circuits Syst. II, Exp. Briefs, vol. 55, no. 2, Feb. 2008, pp. 183-187. 